$15^{\text {th }}$ International Conference on

AEROSPACE SCIENCES \& AVIATION TECHNOLOGY,

ASAT - 15 - May 28 - 30, 2013, Email: asat@mtc.edu.eg,

Military Technical College, Kobry Elkobbah, Cairo, Egypt,

Tel: +(202) $24025292-24036138$, Fax: + +(202) 22621908

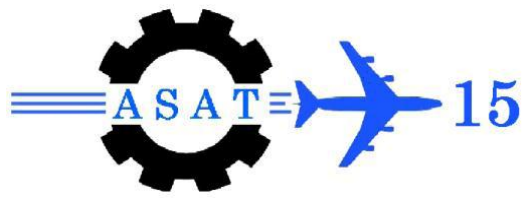

\title{
Drag Braking of the Deployment Mechanism of Solar Array on a Small Satellite
}

\author{
M. G. El-Sherbiny ${ }^{*}$, A. A. Khattab ${ }^{*}$, M. K. Kassab ${ }^{\dagger}$
}

\begin{abstract}
This paper demonstrates Mechanical Desktop modelling and Finite Element Analysis of shoe brake specially designed to apply drag braking on the deployment mechanism of solar array mounted on a small satellite. Strength analysis was performed under realistic loads to the brake assembly, and the most critical points are checked. Several finite element analysis models were considered to verify the integrity of the deployment mechanism. These analyses considered the static, modal and random vibration testing to verify the safe performance of the brake after orbit insertion.
\end{abstract}

Keywords: Satellite, Deployment Mechanism, Brake Shoe, Solar Array, finite Element Analysis, Modal analysis, Random analysis.

\section{Introduction}

Today's engineering design needs to manage the conflicting goals, improving performance while reducing developing time and costs. In this context designers utilized the solar energy as clean renewable source for powering small satellites. Solar arrays of considerable surface area are required to provide enough power for the safe payload functioning and for the computer and the communication systems, [1]. Figure 1 shows a schematic drawing of the satellite with the solar arrays.

Innovative designs included foldable solar arrays to minimize size and space requirement on the launching vehicle. Self-actuated deployment mechanisms utilized the stored energy in a torsion spring to drive the solar arrays during the unfolding phase after orbital insertion. In such cases the motion has to be controlled by drag braking to reduce or eliminate the shock loading at the end of the stroke. Drag brake should be of minimum size and weight but can absorb and dissipate energy enough to make gradual deployment and smooth motion until the mechanism gets to rest at the end of the stroke, without shock loadings or reactions. A special small drum brake, with frictional shoes which are forced in contact with the drum under a compressive spring force, was designed for this particular purpose [3]. Figure 2 shows an isometric drawing of the brake assembly.

Prof. of Machine Design and Engineering Tribology, Cairo University, Egypt.

$\dagger$ Head of Structure Department, National authority for Remote Sensing and Space Sciences (NARSS), Egypt. 


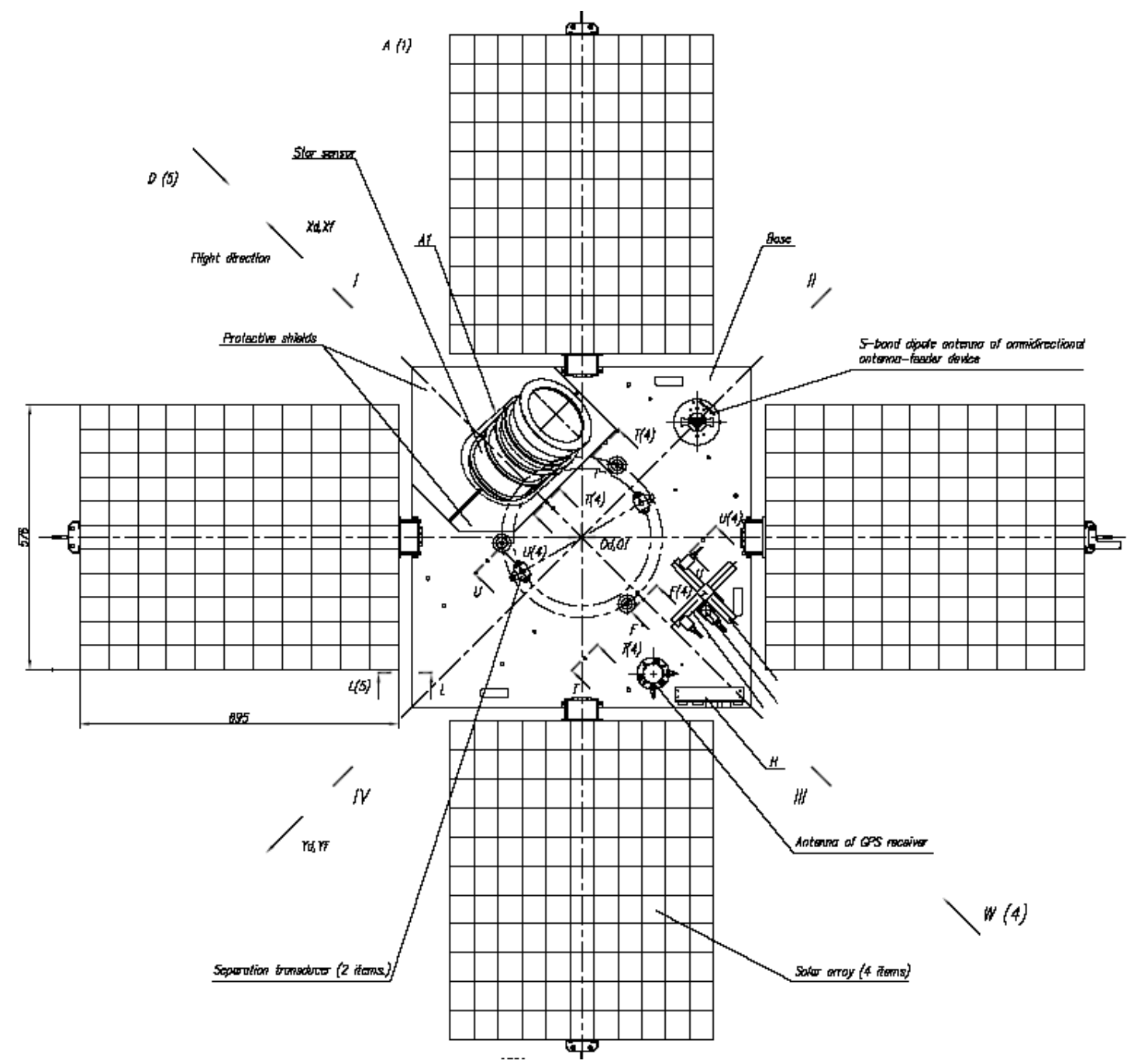

Figure (1): Top View of Small Satellite with Foldable Solar Arrays [2]

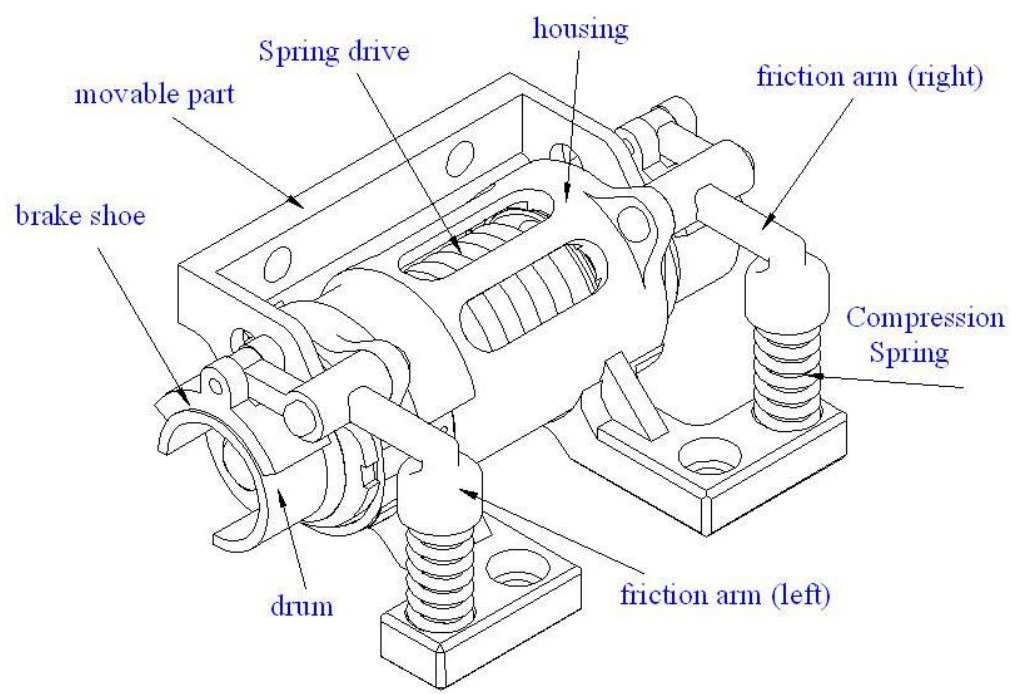

Figure (2): Isometric drawing of the Drag brake of the Deployment Mechanism. 
The brake had an estimated weight of $0.350 \mathrm{Kg}$ and is able to deploy the solar arrays in 2 to 3 seconds depending on the realized coefficient of friction of 0.5 in normal atmosphere and 1.0 in space environment at an altitude of $768 \mathrm{Km}$.

The designed brake has to be checked and tested accurately before the prototype is made available [3]. In the present work the DM is modelled by using Mechanical Desktop (MDT) software and then exported to Finite Element Analysis Package (ANSYS 11). Simulation analysis was made to describe the dynamic behavior of the mechanism structure and to predict its natural frequencies and structure response to the worst case of Launch Vehicle (LV) loads through some of simulation analysis such as static, modal and random vibration analysis [5].

Mechanical structure can resonate, where small forces can result in significant deformation, and damage can be induced in the structure. Resonant vibration is mainly caused by an interaction between inertial and elastic properties of the materials within a structure. To better understand structural vibration problems, the resonant frequencies of the structure have to be identified. Modal analysis is recognized as a technique in finding the modes of vibration of machines, and mechanisms [6].

\section{Structure and Model Description}

The DM is intended for fastening of solar panel to the satellite body, turning the solar panel into the working position and keeping it in this position during operation in orbit. Once the Satellite is in orbit, the Solar Arrays are deployed to function. Because of that, Mathematical Model of DM is constructed using finite Element Computer Package (ANSYS 11). Figure 3 shows the MDT model of the designed DM brake assembly to eject the folded SA and to control its deployment under drag braking. The finite element package provides a library of structure elements, such as beams, shells, and solid elements. The DM is modeled by connecting elements together within the geometrical constraints, material properties, and appropriate boundary conditions to describe the physical constraints. Details of geometry and boundary conditions are discussed in the next sections.

Forces and moments obtained from solar array analysis are given by satellite structure strength analysis $[3,5]$.

$\mathrm{F}_{\mathrm{x}}=-1.291 \times 10^{-2} \mathrm{~N}, \quad \mathrm{~F}_{\mathrm{y}}=-5.33 \times 10^{-4} \mathrm{~N}, \quad \mathrm{~F}_{\mathrm{z}}=-1.651 \times 10^{-2} \mathrm{~N}$. $\mathrm{M}_{\mathrm{x}}=7.28 \times 10^{-3} \mathrm{Nm}, \quad \mathrm{M}_{\mathrm{y}}=-5.795 \times 10^{-3} \mathrm{Nm} \quad \mathrm{M}_{\mathrm{z}}=5.745 \times 10^{-3} \mathrm{Nm}$.

FE analysis of DM is performed to make sure that the DM structure will withstand the loads at the stoppage point. It is recommended to run modal analysis to make sure the natural frequencies of SA will not coincide with the DM ones.

ANSYS 11 FE package is used to make the design analysis and verify the design [7]. The method uses a complex system of points (nodes) which form a grid called a mesh. Fine mesh is used to model fine geometrical details as well as closely approximate the localized stresses and strains in areas of steep gradients or of high stress concentrations. The model represents the assembly of all components and parts of the mechanism linked together and exposed to maximum loading conditions. 


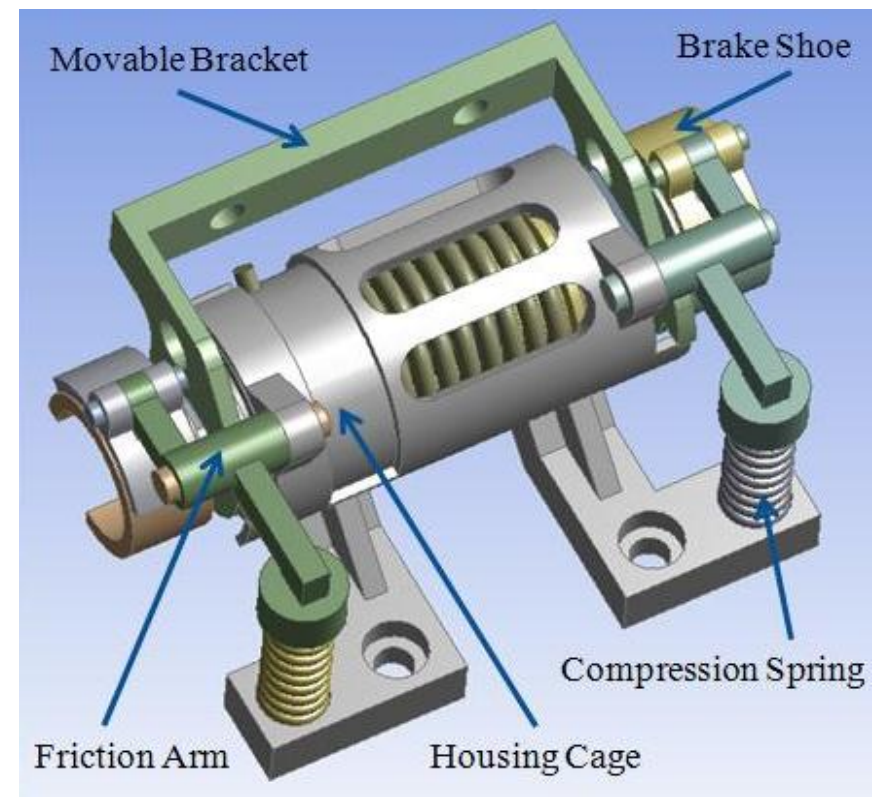

Figure (3): Isometric View for Deployment Mechanism.

\section{Mesh Generation}

Tetrahedral elements are used as shown in fig. (4). When using high relevance is 100 , and relevance center is fine, the meshing was somewhat difficult. But when simplifying relevance to 60 or 50 and relevance center to medium or coarse, the meshing problem was smoothly solved.

Using relevance of 100; and relevance center medium, one gets:

No. of elements: 595160;

No. of degrees of freedom (No. of Nodes): 1249356.

Whilst using relevance of 50; and relevance center medium, gives:

No. of elements was: $\mathbf{9 0 9 1 4}$;

No. of degrees of freedom (No. of Nodes): 194640,

And therefore the latter case is more appropriate to minimize truncations and round off errors. Figure 2 shows the mesh design of the DM brake assembly.

\section{Materials}

The materials were selected to meet the requirements of each individual component. Structural steel was selected for parts: rotating shaft, screws, and Joints. AISI 4130 steels and 304 stainless steels were used for drums and ball bearings, Gray cast iron is also used for brake shoes, Aluminum alloy AMG6 is used for movable bracket, housing cage and friction arms, whilst other commercial aluminum alloys were used for guiding bushes, Piano wire spring DIN 17223D is used for spring elements. The mechanical, thermal and physical properties of these materials are given in the Appendix. 


\section{Static Analysis}

The objective of static loading is to define the resulting load distribution, strains and stresses throughout the structure of mechanism components. When performing static analysis with load factors, forces and moments are applied to the assembly of the mechanism components. The linear static analysis in ANSYS is used to compute stresses, strains and deformations [8].

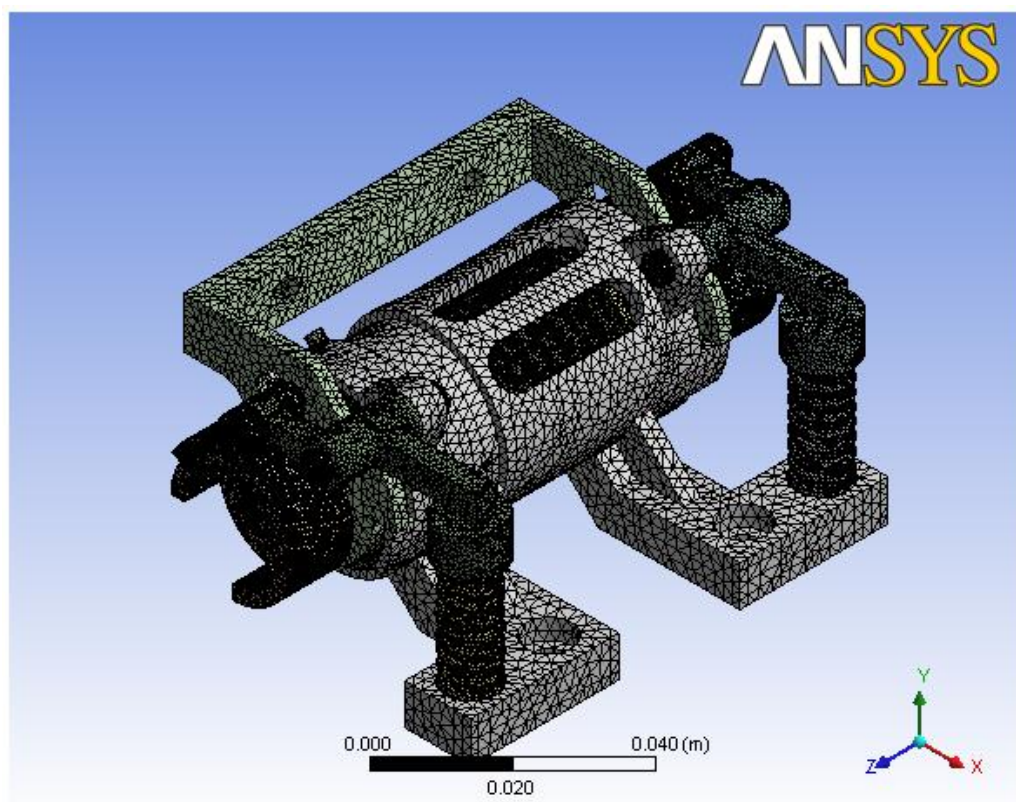

Figure (4): Mesh of Deployment Mechanism.

Figure (5) represents the ANSYS static analysis from geometry definition passing through connection definition, meshing the model and up to post processing of the static structural results. It also shows the critical areas of the maximum deformation which occurs on the movable bracket and this maximum computed value as 2,2501e-004 $\mathrm{m}$. It is obvious that deformations are small and will not affect the structure of SA and mechanism movable bracket.

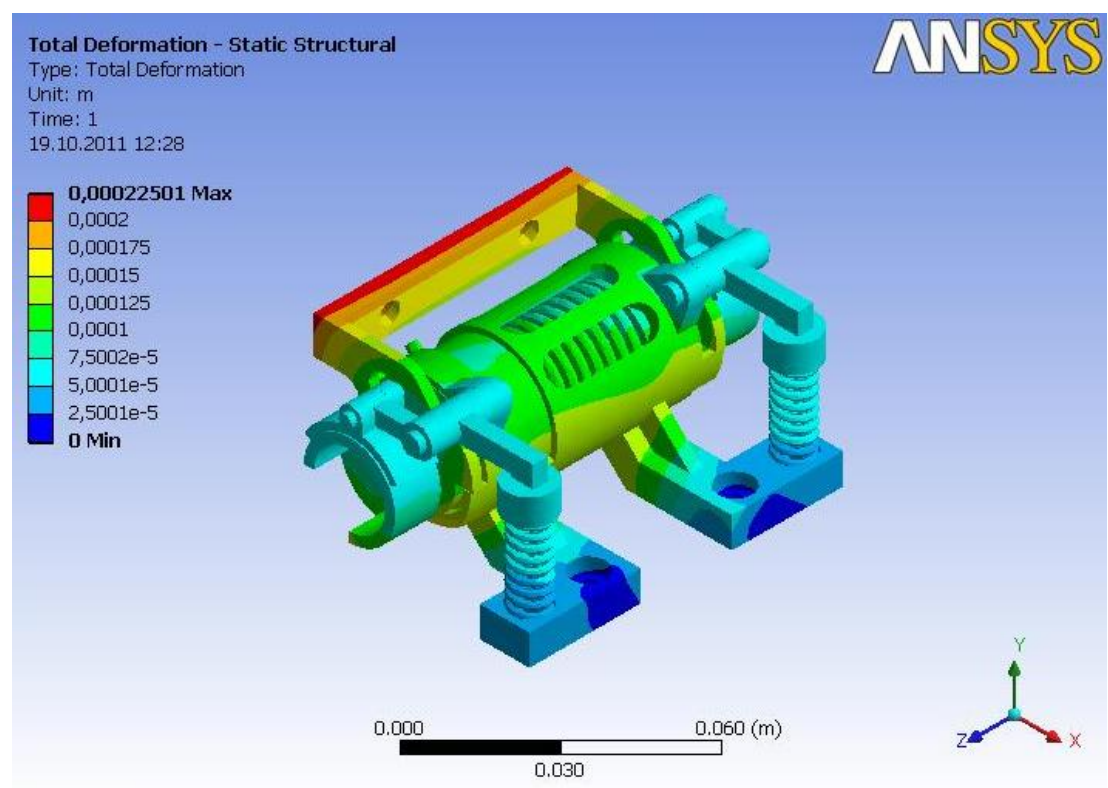

Figure (5): Total Deformation of Static Analysis of DM. 
Figure (6) shows the critical areas of the highest strain in the DM, and also shows the locations of applied loads and the fixed nodal points resembling fixed supports. From analysis it was found that the most critical strain occurs on housing cage $2,1718 \mathrm{e}-002 \mathrm{~m} / \mathrm{m}$.

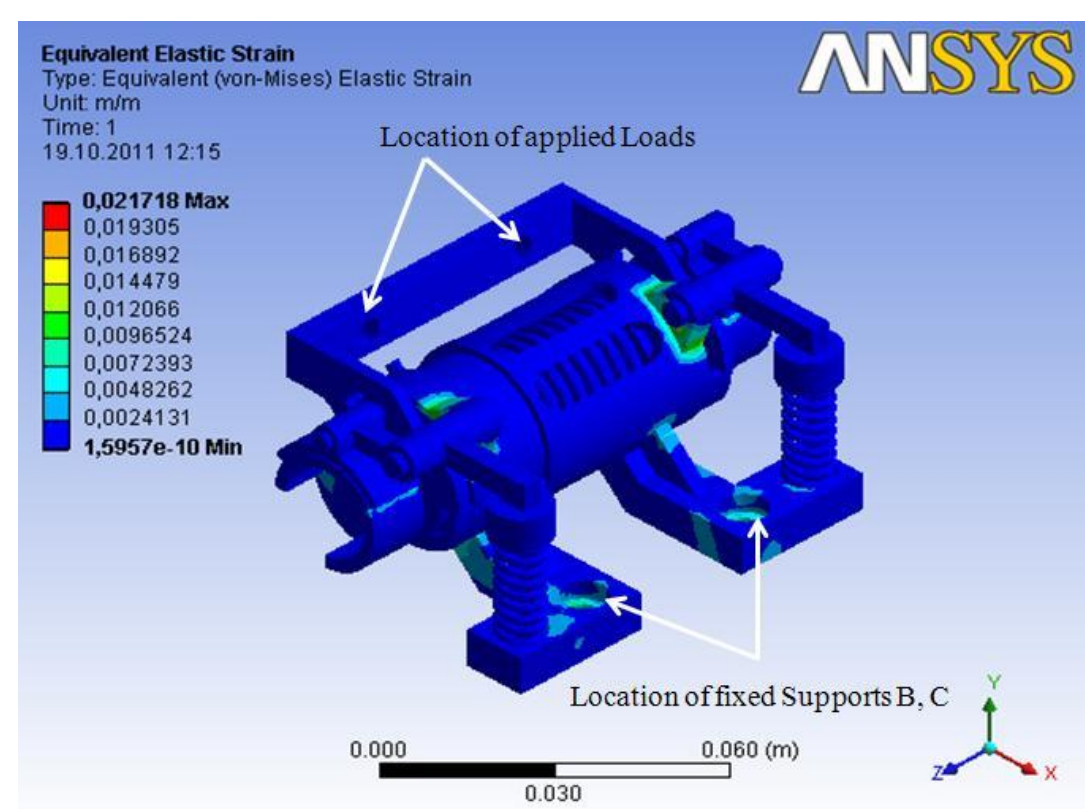

Figure (6): Distribution of Equivalent Elastic Strains (Von-Mises) on DM.

Figure (7) shows the critical regions or the most stressed regions in the DM. From analysis it was found that the most critical stress occurs on cubic joint and its value is $2,7544 \mathrm{e}+008 \mathrm{~Pa}$. Obviously the computed max shear stresses were far below the shear strength of the part and therefore the design was mechanically safe.

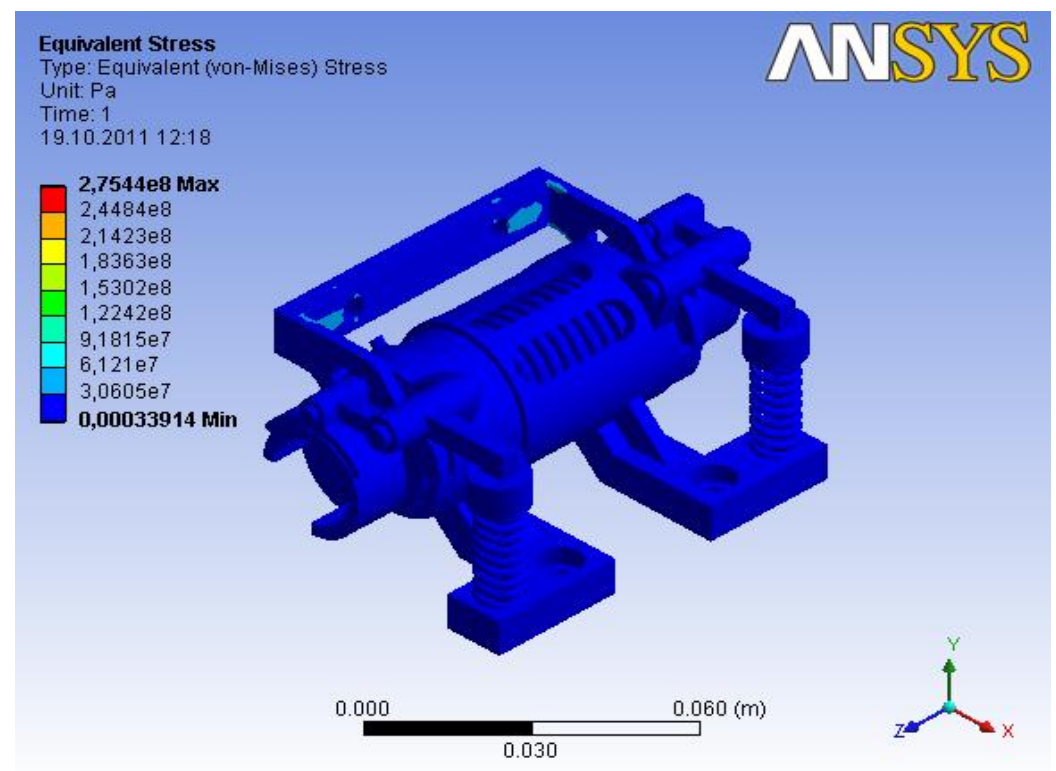

Figure (7): Distribution of Equivalent Stresses (Von-Mises) on DM. 


\section{Modal Analysis of DM}

Modal analysis and testing is used to identify the vibration modes and their natural frequencies, and to provide the structural matrices, which is required for the dynamic analysis of the assembly. Thus the basic structural dynamic data, when obtained accurately from a valid test also provides a true identification of the structural behavior at the modes of interest. These derived matrices are based on the measured contributions of the mass, stiffness and damping properties at the modes of interest, taking into considerations the actual boundary conditions. These data is then used in a finite element model of the structure, for subsequent problem solving, or re-designing the mechanism for better dynamic response $[9,10]$.

Modal analysis was performed to the model of DM using ANSYS 11 software and the model has been solved with first 5 modes. Figures 8 to 12 show the resulting first five modes of vibration. This analysis is also used to gain better understanding of DM behavior and response to environmental conditions which of course, lead to better planning of the experimental testing.

The normal modes obtained from the FEA for DM model before testing, represent fairly reasonable estimation of the DM Eigen frequencies and mode shapes. This information is used to plan the real testing; that is, to determine excitation conditions, shaker locations and accelerometer locations.

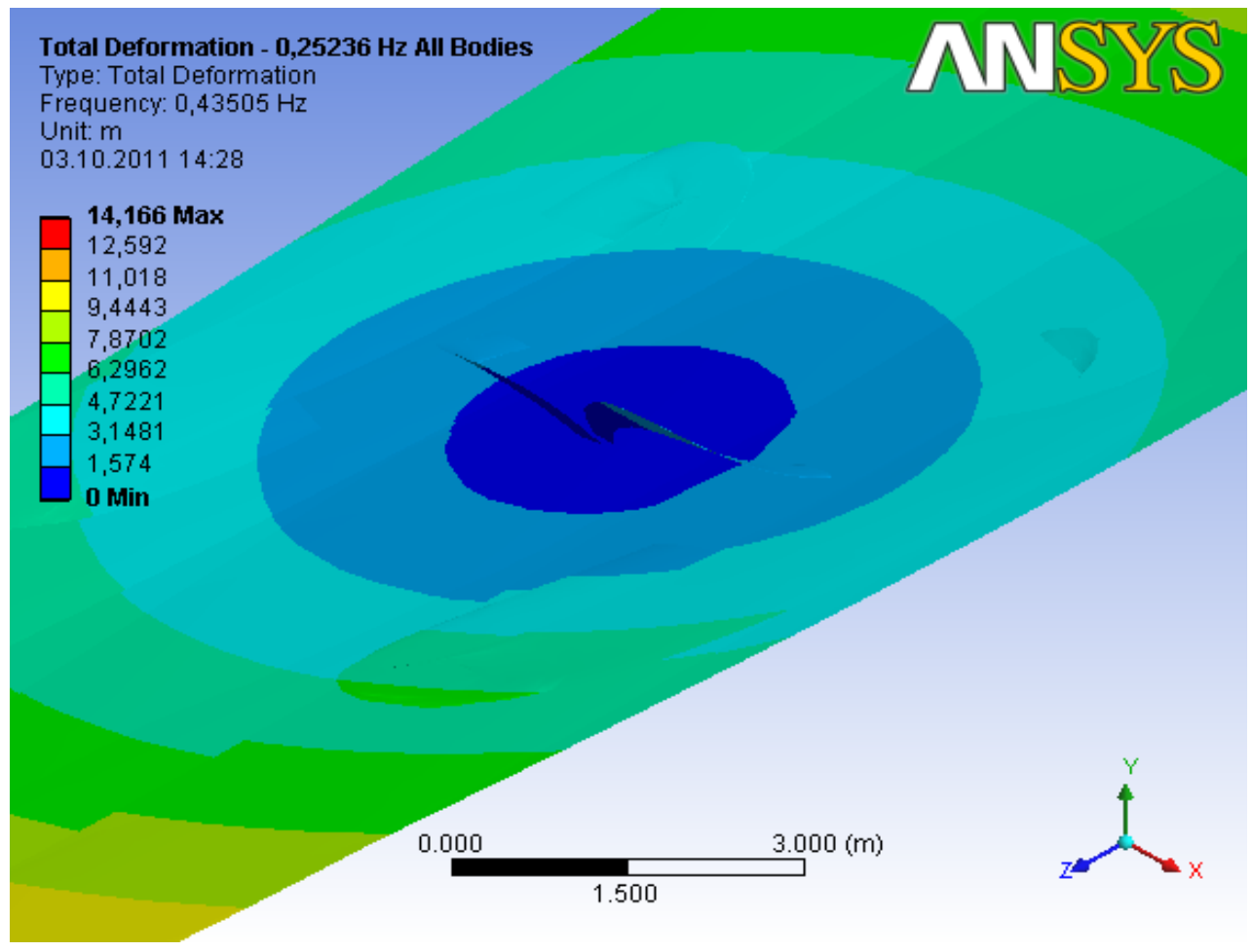

Figure (8): 1st mode shape of DM structure at $f n=0.25236 \mathrm{~Hz}$. 


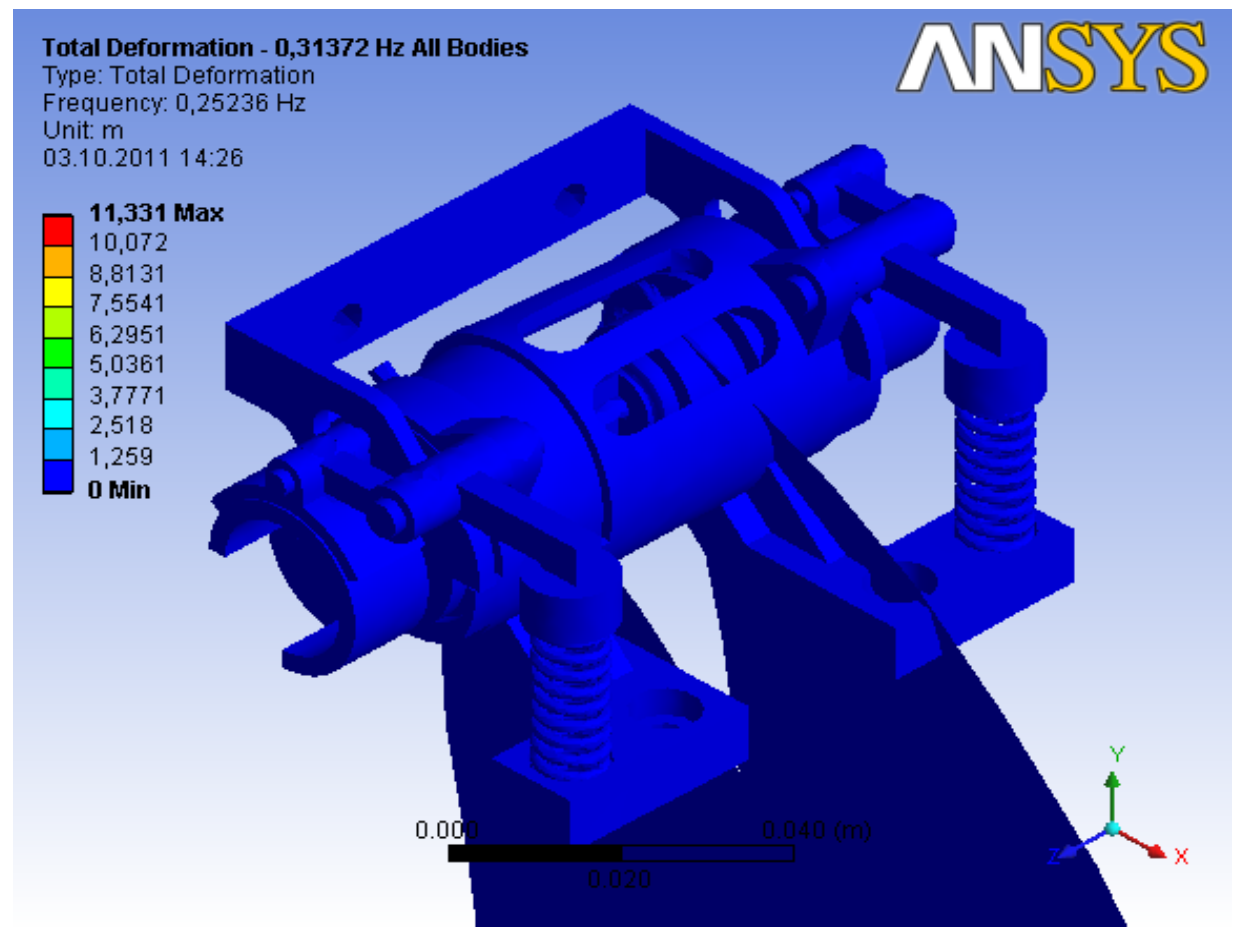

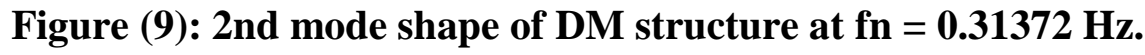

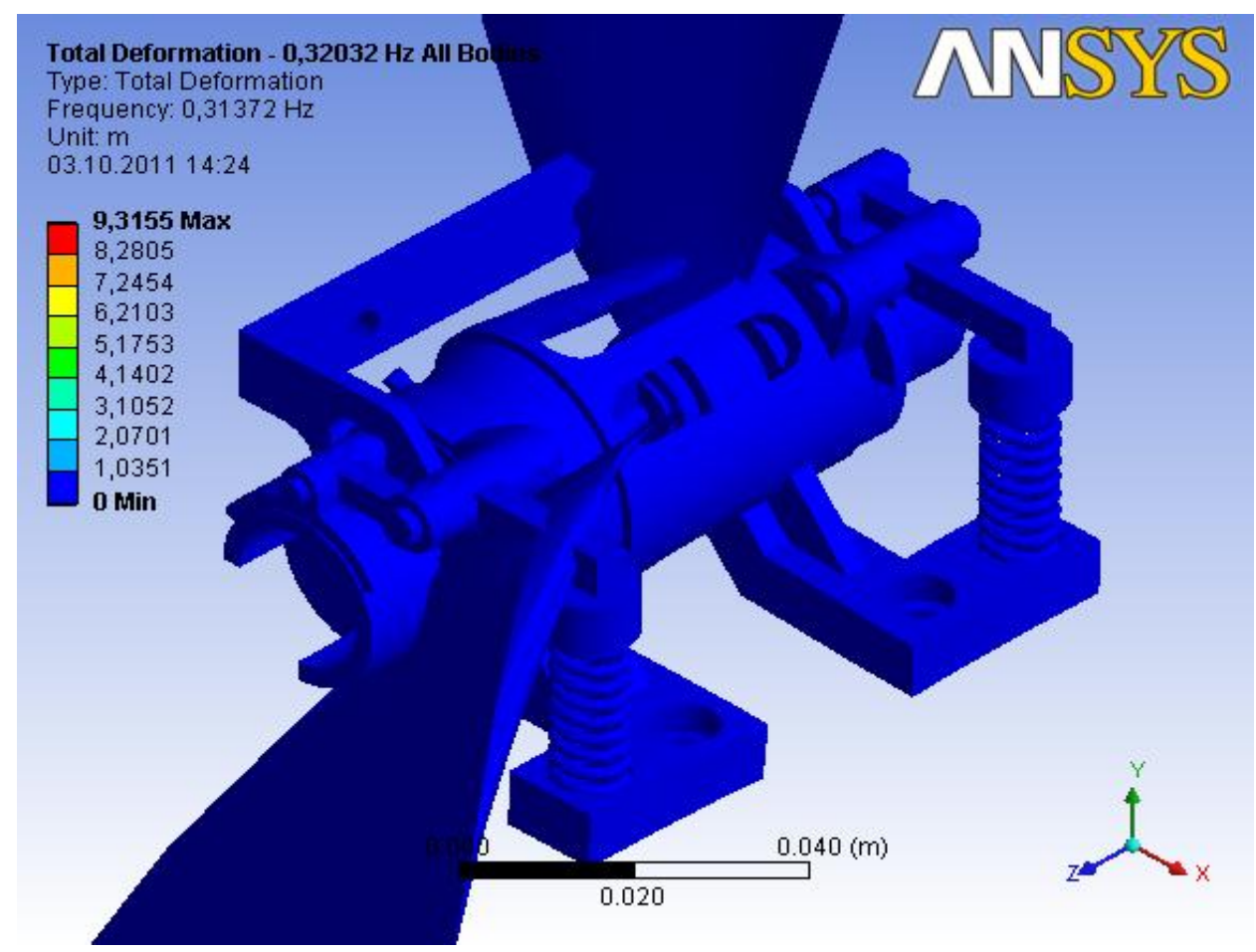

Figure (6): 3rd mode shape of DM structure at $f_{n}=0.32032 \mathrm{~Hz}$. 


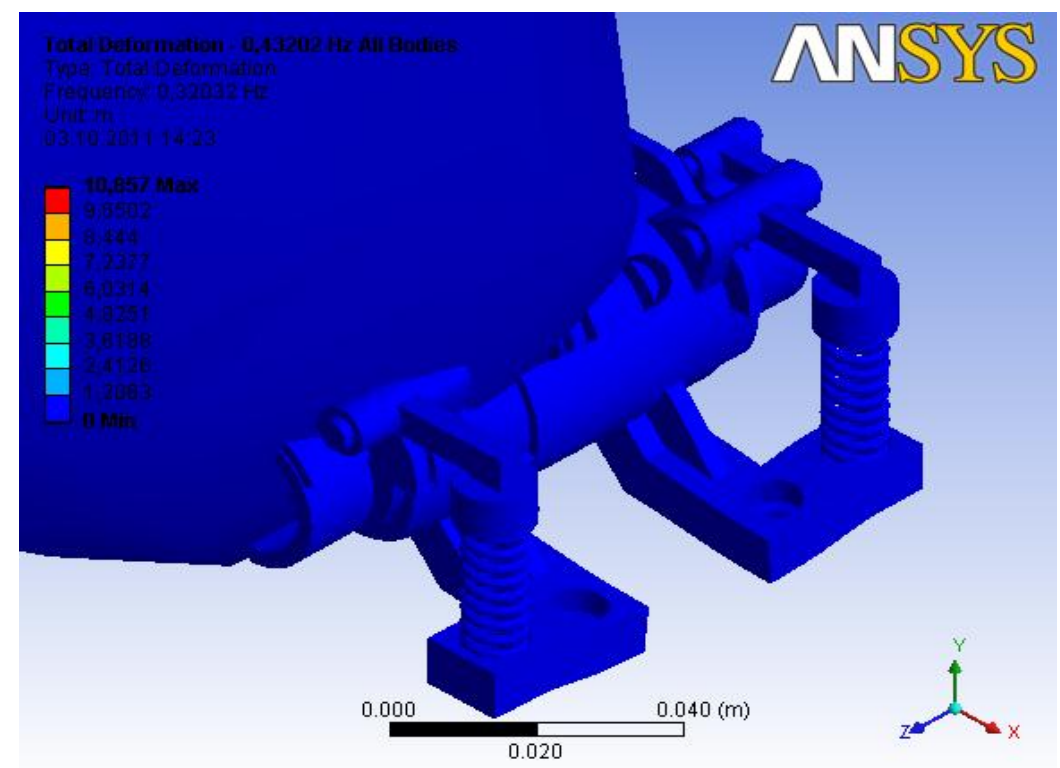

Figure (7): $4^{\text {th }}$ mode shape of DM at $f_{n}=0.43202 \mathrm{~Hz}$.

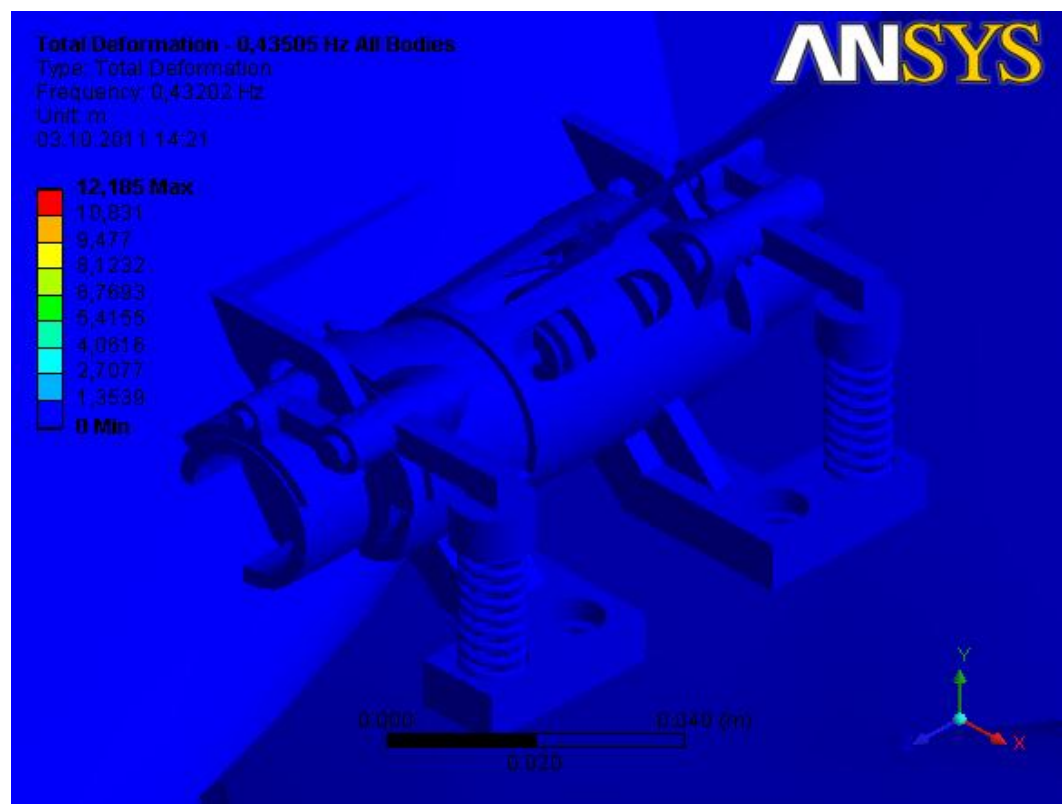

Figure (8): $5^{\text {th }}$ mode shape of DM structure at $f_{n}=0.43505 ~ H z$.

\section{Random Analysis}

The fundamental nature of random vibration are needed to verify design, develop and produce cost effective and lightweight mechanisms that are capable of operating in various environments with high degree of reliability. The characteristic of random vibration is nonperiodic and it can be considered as a series of overlapping sinusoidal curves [4]. In this environment all the exciting frequencies within a given bandwidth are excited at the same time.

One of the different types of curves that can be used to show the random vibration input requirements is the Power Spectral Density (PSD) curve Table (1) shows the PSD data. This 
is also shown in log-log scale with power spectral density $\left(\mathrm{G}^{2} / \mathrm{Hz}\right)$ along the ordinate vertical axis and frequency $(\mathrm{Hz})$ along the horizontal abscissa axis. It should be noted that acceleration is represented as root mean square (RMS) and it is the area under the random vibration curve. Figure (13) shows the shaped random vibration input curve for the DM model.

Table (1): PSD Acceleration vs. Frequency.

\begin{tabular}{c|c}
\hline Frequency $\mathrm{Hz}$ & PSD Acceleration $\left(\mathrm{m} / \mathrm{sec}^{2}\right)^{2} / \mathrm{Hz}$ \\
\hline 10,0 & 1,0 \\
\hline 25,0 & 1,3 \\
\hline 50,0 & 1,0 \\
\hline 80,0 & 3,1 \\
\hline 130,0 & 1,6 \\
\hline 160,0 & 2,8 \\
\hline 190,0 & 1,2 \\
\hline 225,0 & 2,0 \\
\hline 270,0 & 2,8 \\
\hline 310,0 & 5,0 \\
\hline \hline
\end{tabular}

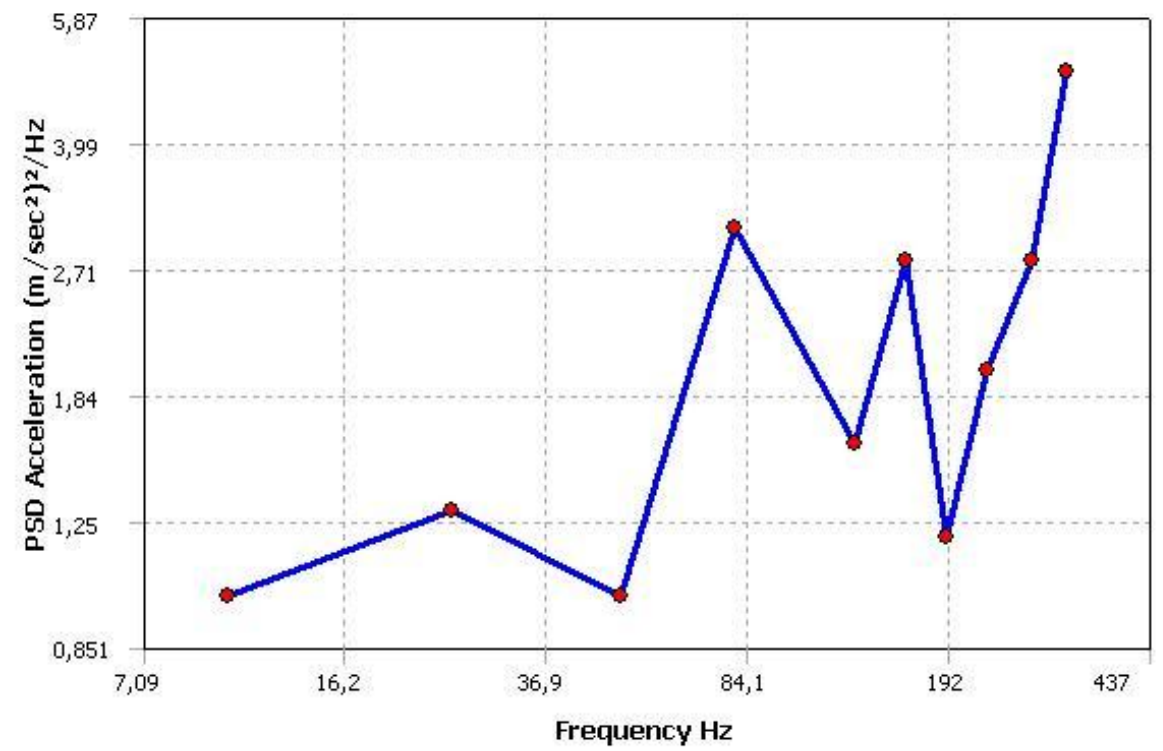

Figure (9): PSD Acceleration vs. Frequency.

Figure (14) shows the resulting stresses of DM analysis and it is seen that stress distribution values have occurred at scale factor 1 sigma, and probability of $68.3 \%$ of the time at X-axis direction. It also, shows that the maximum effect of frequencies occurs on Torsion Spring and compression spring with a value of $733.18 \mathrm{~Pa}$. These stresses do not form any threatening for DM and it can be said that DM can withstand Vibration loads well after an orbit insertion of the satellite. 


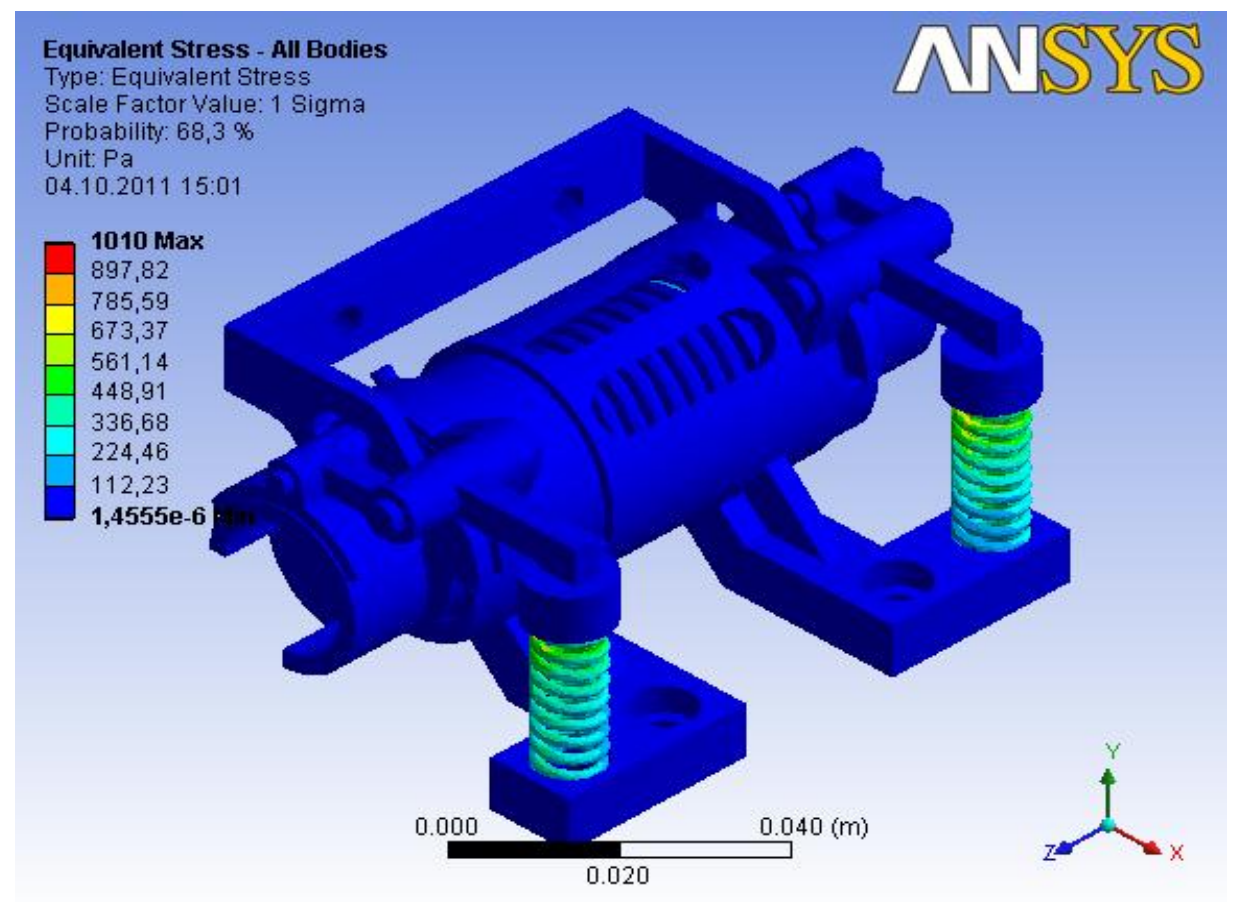

Figure (10): Stress Distribution on DM components.

Figures (15 to 23) shows that deformations occurred under the effect of frequencies load at all directions (X, Y, and $\mathrm{Z}$ ) and the resulting deformations due to velocity and acceleration loads do not represent any threatening to any of the DM components during satellite operation after an orbit insertion.

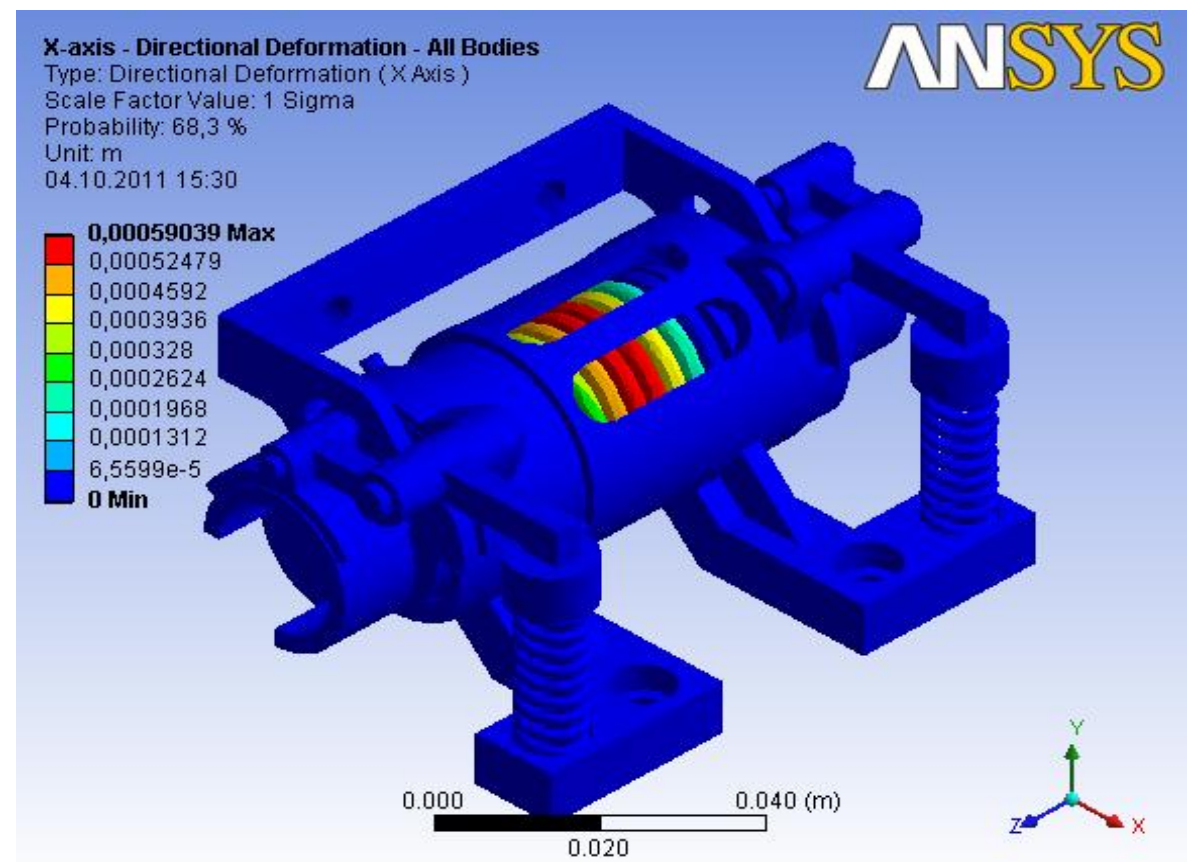

Figure (11): Direction Deformation on DM components at $\mathrm{X}$-axis. 


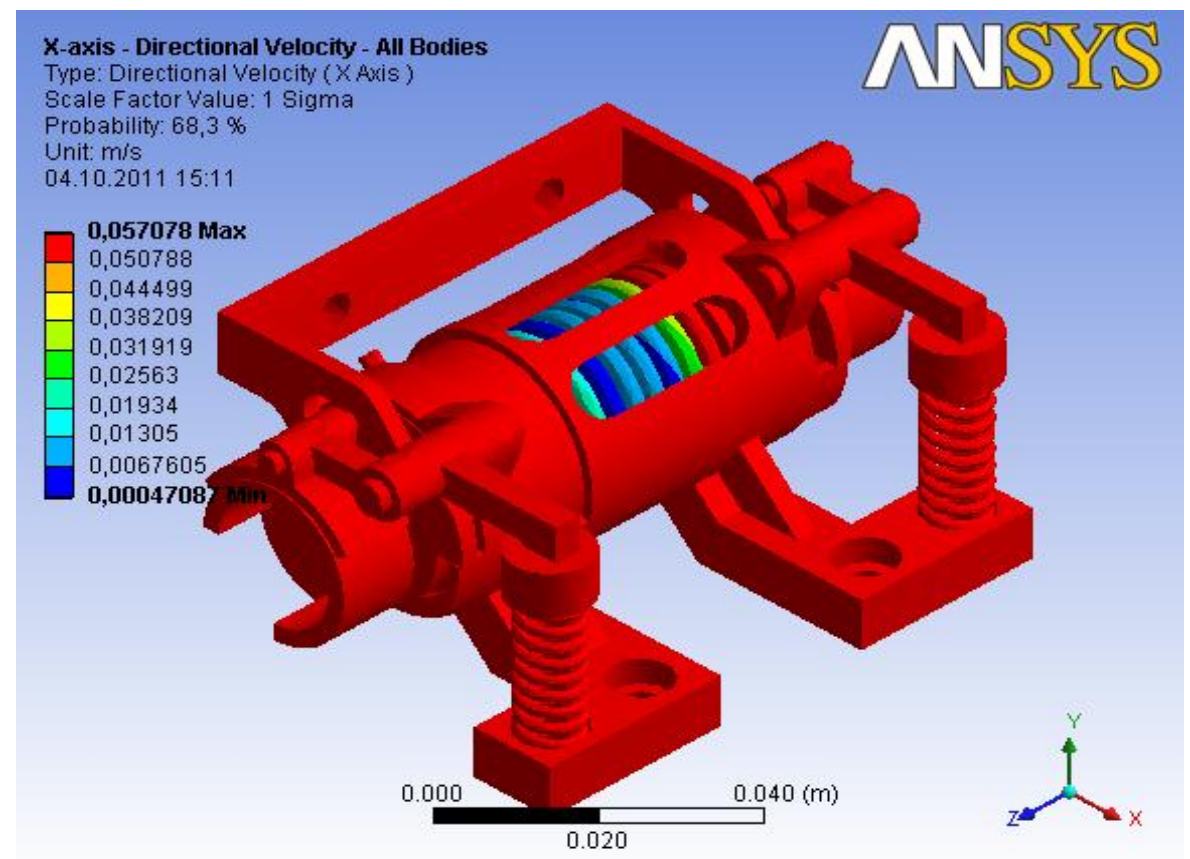

Figure (12): Velocity Deformation Distribution on $\mathrm{DM}$ components at $\mathrm{X}$-axis.

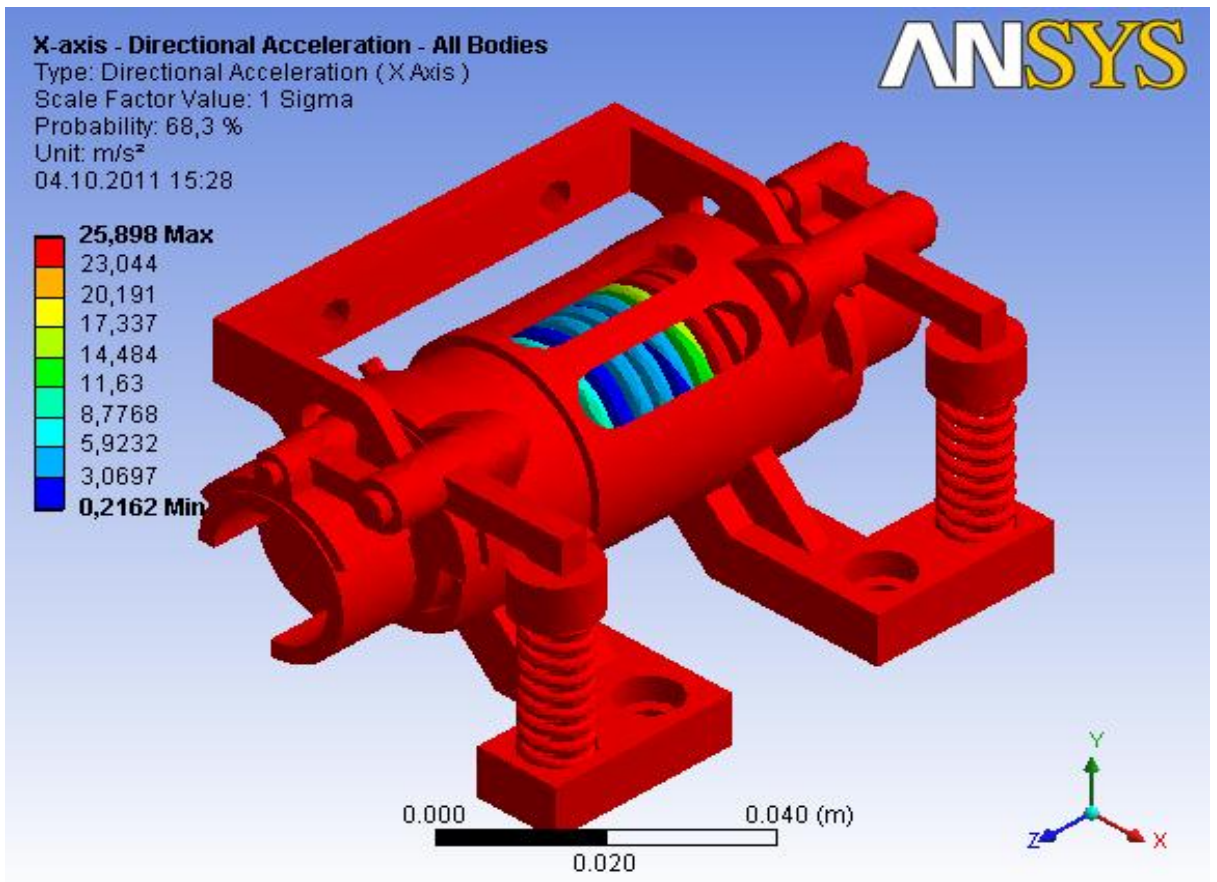

Figure (13): Acceleration Deformation Distribution on DM components at $\mathrm{X}$-axis. 


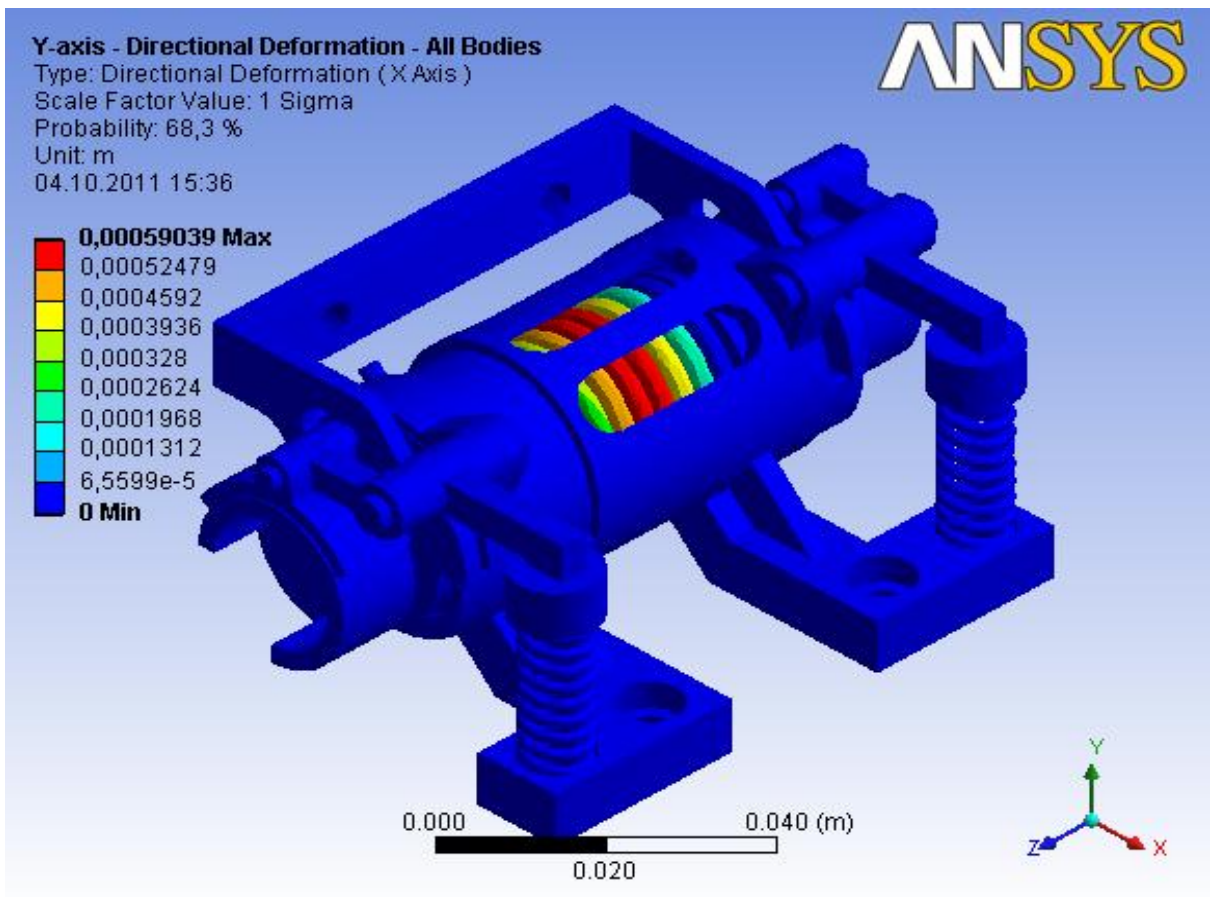

Figure (14): Directional Deformation Distribution on DM components at $\mathrm{Y}$-axis.

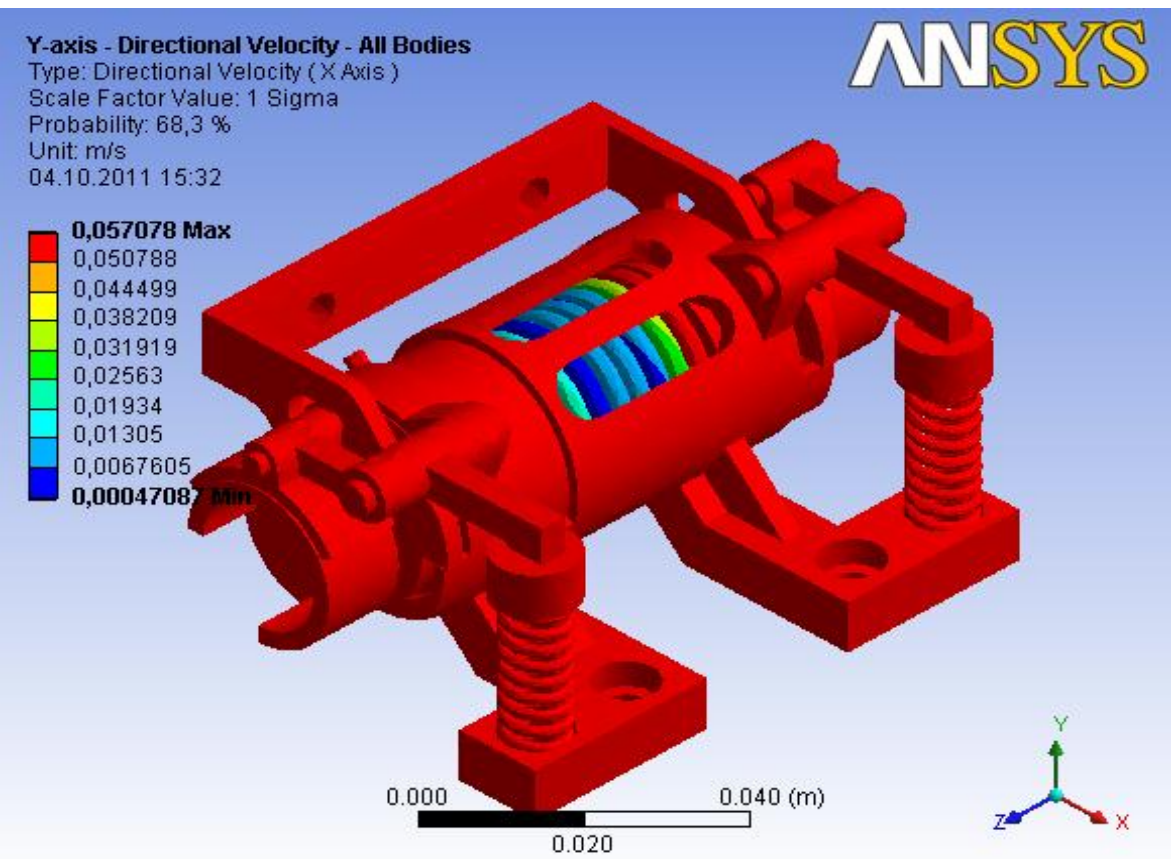

Figure (15): Velocity Deformation Distribution on DM components at $Y$-axis. 


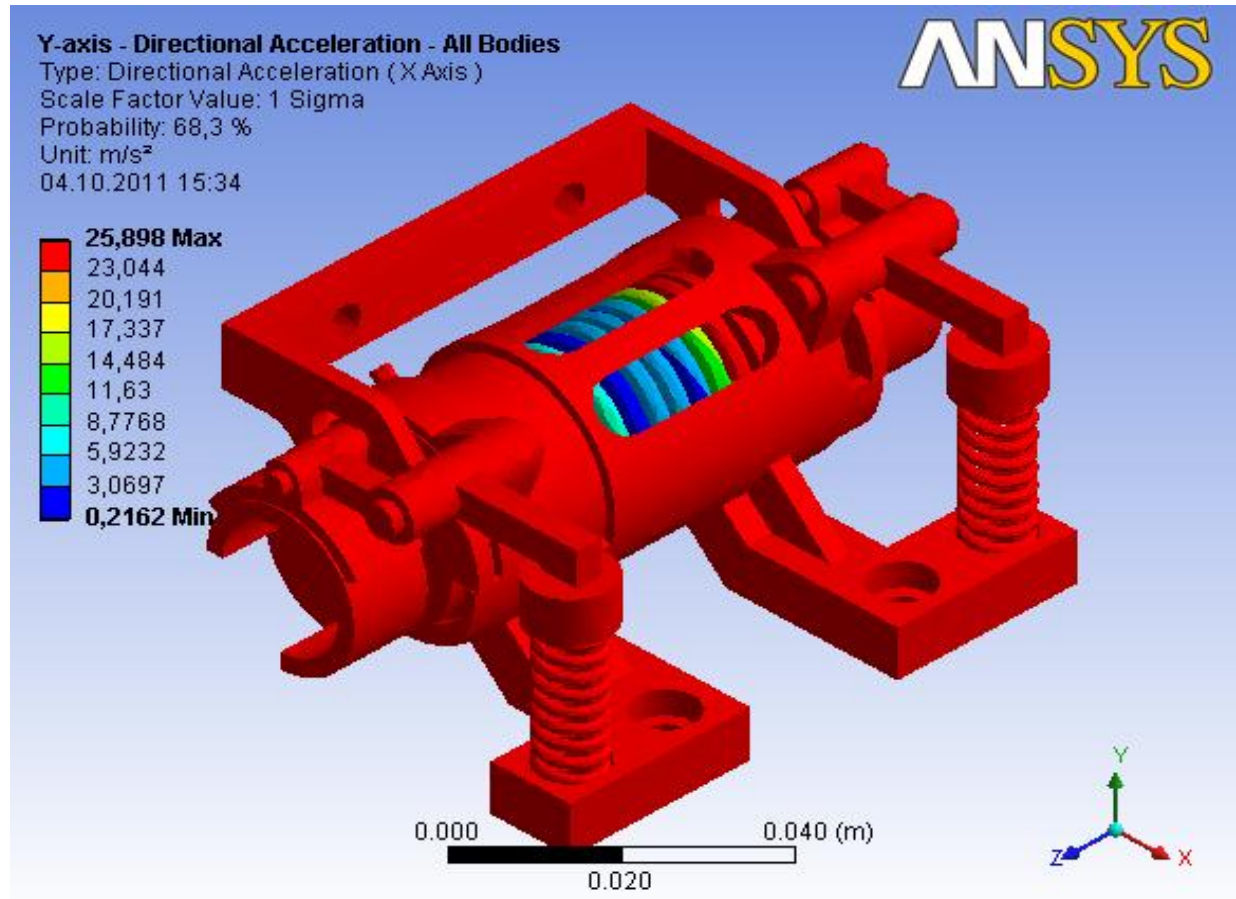

Figure (16): Acceleration Deformation Distribution on DM components at Y-axis.

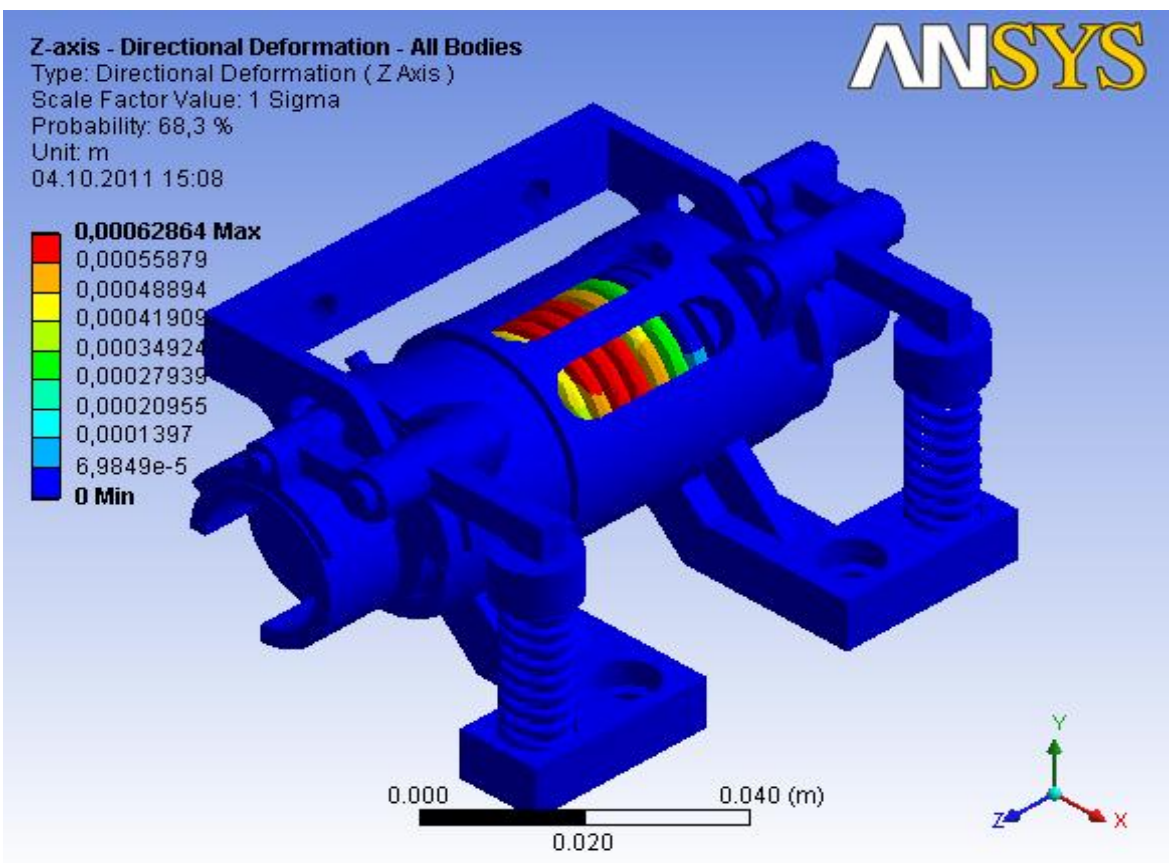

Figure (17): Directional Deformation Distribution on DM components at $\mathrm{Z}$-axis. 


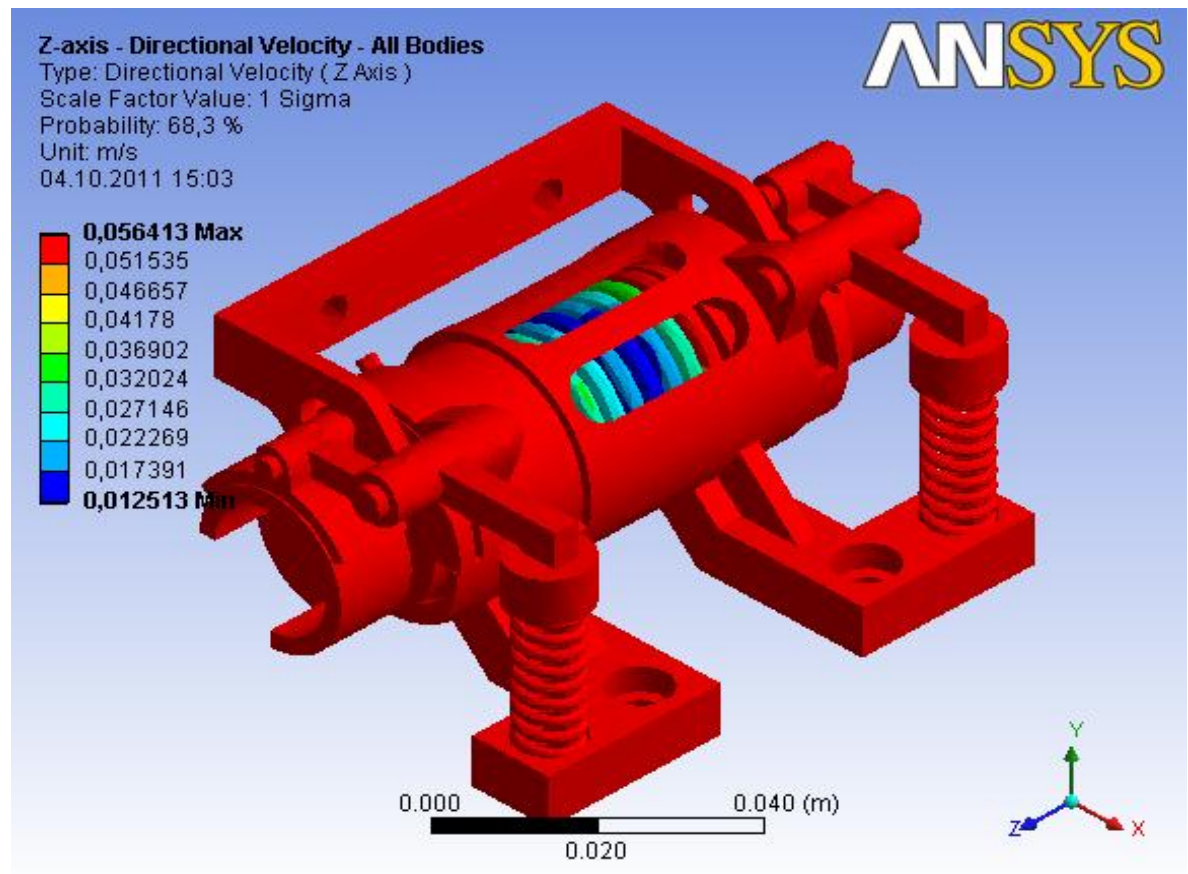

Figure (18): Velocity Deformation Distribution on DM components at $\mathrm{Z}$-axis.

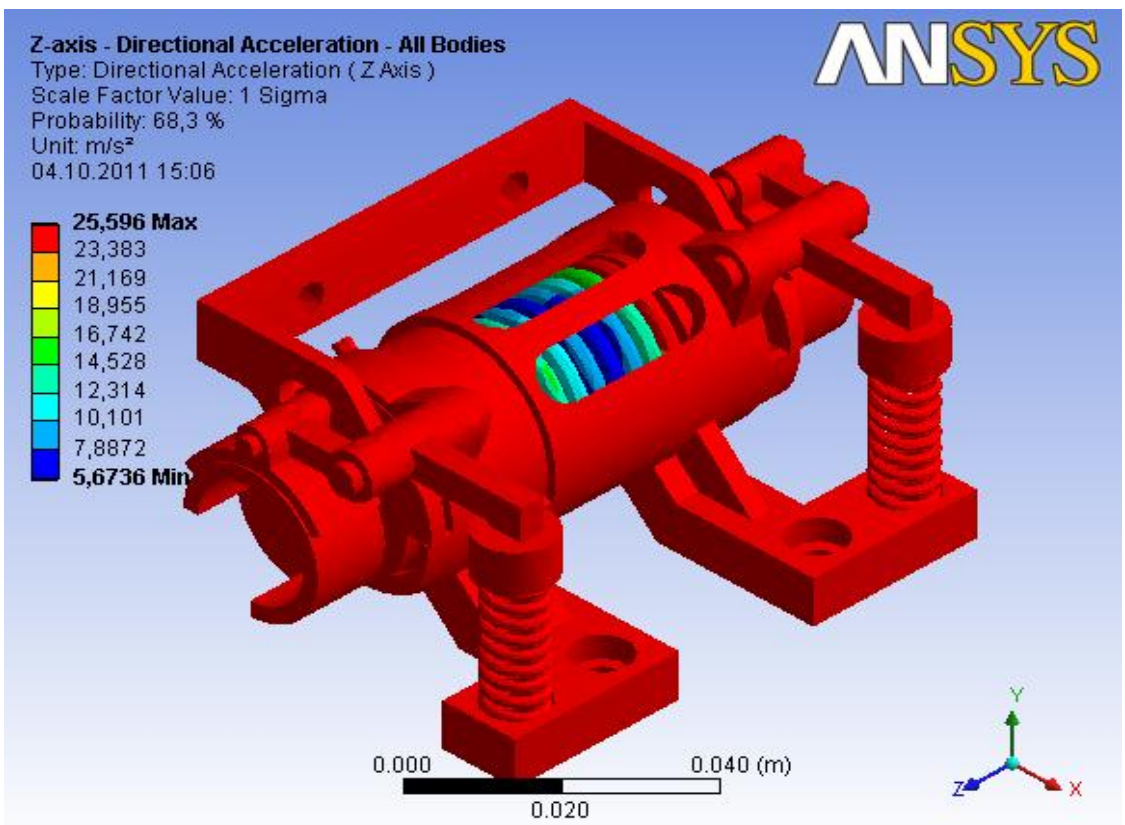

Figure (19): Acceleration Deformation Distribution on DM components at $\mathrm{Z}$-axis.

\section{Conclusion}

Linear static analysis of the DM was performed using FE analysis on ANSYS software and the deformation and displacement are calculated. The modal analysis was performed and the first five resonance frequencies (mode shapes) are calculated. Random vibration analysis was also performed and the results revealed that the maximum stresses occurred on Torsion 
Spring and Compression Spring with a value of $733.18 \mathrm{~Pa}$. one therefore can conclude that the FE analysis of the proposed design of the DM for SA of a small satellite showed that the model survived vibration loads, moments, and forces at operating conditions similar to those expected after an orbit insertion of the satellite.

\section{References}

[1] Heylen W., Lammens S., Sas P.,: Modal Analysis Theory and Testing, Katholieke Universiteit, Leuven, 1997.

[2] EgyptSat-1 Project, Satellite Preliminary Design. Album of Drawings. Doc. No. EGS YZH RPT 01801.

[3] Kassab, M. "Design, Analysis, Manufacturing, and Testing of a Deployment Mechanism for Solar Panel of a Small Satellite"; Ph.D. thesis, Faculty of Engineering, Cairo University, Egypt, December 2011.

[4] Friswell, M. I., Mottershead J. E., “ Finite Element Model Updating in Structural Dynamics" Dept. of Mechanical Engineering, University of Swansea, Swansea, U.K. 1996.

[5] Shibabrat N., Wrik M., "Experimental Modal Testing for Estimating the Dynamic Properties of a Cantilever Beam" Department of Civil Engineering , Jadavpur University, Kolkato-700 032.

[6] Bart B. Wim H. and Jan D.; "Modern Solutions for Ground Vibration Testing of Large Aircraft"; LMS International, Leuven, Belgium.

[7] EgyptSat-1 Project, Satellite Preliminary Design. Composition and main Characteristics, Doc. No.EGS YZH RPT 01801.

[8] ANSYS, Inc. Release 11.0 Documentaion for ANSYS.

[9] Sudharsan, M; "Structural Design and Analysis of a lightweight Composite Sandwich Space Radiator Panel" Msc. Thesis, Office of Graduate Studies of Texas A\&M University, USA, December 2003.

[10] Neville F. Rieger; "the Relation between Finite Element Analysis and Modal Analysis"; Stress Technology Incorporated, Rochester, New York.

Appendix

Mechanical, Thermal and Physical properties of Used Materials

\begin{tabular}{c|c|c|c|c|c|c|c}
\hline \hline Property & $\begin{array}{c}\text { Young's } \\
\text { Modulus } \\
{[\mathrm{Pa}]}\end{array}$ & $\begin{array}{c}\text { Poisson's } \\
\text { Ratio }\end{array}$ & $\begin{array}{c}\text { Density } \\
{\left[\mathrm{kg} / \mathrm{m}^{3}\right]}\end{array}$ & $\begin{array}{c}\text { Tensile } \\
\text { Yield } \\
\text { Strength }[\mathrm{Pa}]\end{array}$ & $\begin{array}{c}\text { Tensile } \\
\text { Ultimate } \\
\text { Strength }[\mathrm{Pa}]\end{array}$ & $\begin{array}{c}\text { Thermal } \\
\text { Conductivity } \\
{\left[\mathrm{W} / \mathrm{m}-{ }^{\circ} \mathrm{C}\right]}\end{array}$ & $\begin{array}{c}\text { Specific } \\
\text { Heat, } \\
{\left[\mathrm{J} / \mathrm{kg}-{ }^{\circ} \mathrm{C}\right]}\end{array}$ \\
\hline $\begin{array}{c}\text { Structural } \\
\text { Steel }\end{array}$ & $2.00 \times 10^{11}$ & 0.3 & 7850 & $2.50 \times 10^{8}$ & $4.60 \times 10^{8}$ & 60.5 & 434 \\
\hline $\begin{array}{c}\text { Aluminum } \\
\text { alloy }\end{array}$ & $7.10 \times 10^{10}$ & 0.33 & 2770 & $2.80 \times 10^{8}$ & $3.1 \times 10^{8}$ & & 875 \\
\hline $\begin{array}{c}\text { Steel Standard } \\
\text { AISI 4130 }\end{array}$ & $2.00 \times 10^{5}$ & 0.3 & 7700 & & & & 480 \\
\hline $\begin{array}{c}\text { Stainless Steel } \\
\text { Aluminum }\end{array}$ & $1.93 \times 10^{11}$ & 0.31 & 7750 & $2.07 \times 10^{8}$ & $5.86 \times 10^{8}$ & & 875 \\
\hline Alloy AMG6 & $7.10 \times 10^{10}$ & 0.33 & 2770 & & & & 450 \\
\hline $\begin{array}{c}\text { Piano Wire } \\
\text { Steel DIN } \\
\text { 17223D }\end{array}$ & $2.06 \times 10^{11}$ & 0.29 & 7850 & & & & 450 \\
\hline $\begin{array}{c}\text { Gray Cast } \\
\text { Iron }\end{array}$ & $1.10 \times 10^{11}$ & 0.28 & 7200 & $2.40 \times 10^{8}$ & & & 447 \\
\hline \hline
\end{tabular}

